\title{
INTERPOLATION IN VARIABLE EXPONENT SPACES
}

\author{
ALEXANDRE ALMEIDA ${ }^{1}$ AND PETER HÄSTÖ*,2
}

\begin{abstract}
In this paper we study both real and complex interpolation in the recently introduced scales of variable exponent Besov and Triebel-Lizorkin spaces. We also take advantage of some interpolation results to study a trace property and some pseudodifferential operators acting in the variable index Besov scale.
\end{abstract}

\section{INTRODUCTION}

Spaces of variable integrability, also known as variable exponent function spaces, can be traced back to 1931 and W. Orlicz [32], but the modern development started with the paper [27] of Kováčik and Rákosník in 1991. Corresponding PDE with non-standard growth have been studied since the same time. For an overview we refer to the monograph [11] and the survey [19]. Apart from interesting theoretical considerations, the motivation to study such function spaces comes from applications to fluid dynamics [34], image processing [10, 18, 31], PDE and the calculus of variations [1, 2, 14, 15].

In a recent effort to complete the picture of the variable exponent Lebesgue and Sobolev spaces, Almeida and Samko [5] and Gurka, Harjulehto and Nekvinda [17] introduced variable exponent Bessel potential spaces $\mathcal{L}^{\alpha, p(\cdot)}$ with constant $\alpha \in \mathbb{R}$. As in the classical case, this space coincides with the Lebesgue/Sobolev space for integer $\alpha$. A further step was taken by $\mathrm{Xu}[42,43]$, who considered Besov $B_{p(\cdot), q}^{\alpha}$ and Triebel-Lizorkin $F_{p(\cdot), q}^{\alpha}$ spaces with variable $p$, but fixed $q$ and $\alpha$.

Along a different line of inquiry, Leopold $[28,30]$ considered a generalization of Besov spaces where the smoothness index is determined by certain symbols of hypoelliptic pseudo-differential operators. In particular, for symbols of the form $\left(1+|\xi|^{2}\right)^{m(x) / 2}$ the related spaces coincide with the spaces $B_{p, p}^{m(\cdot)}$. Function spaces of variable smoothness have recently been studied by Besov [9]: he generalized Leopold's work by considering both Triebel-Lizorkin spaces $F_{p, q}^{\alpha(\cdot)}$ and Besov spaces $B_{p, q}^{\alpha(\cdot)}$ in $\mathbb{R}^{n}$. By way of application, Schneider, Reichmann and Schwab [35] used $B_{2,2}^{m(\cdot)}(\mathbb{R})$ in the analysis of certain Black-Scholes equations.

Integrating the above mentioned spaces into a single larger scale promises similar gains and simplifications as were seen in the constant exponent case in the $60 \mathrm{~s}$ and $70 \mathrm{~s}$ with the advent of the full Besov and Triebel-Lizorkin scales; however, this requires all the indices to be variable. Thus Diening, Hästö and Roudenko [13] introduced TriebelLizorkin spaces $F_{p(\cdot), q(\cdot)}^{\alpha(\cdot)}$, and we introduced in [3] Besov spaces $B_{p(\cdot), q(\cdot)}^{\alpha(\cdot)}$ with all three indices variable. Additional results, including the Sobolev embedding, have subsequently been proven by Kempka and Vybíral [21, 22, 23, 24, 41].

Date: September 6, 2013.

2000 Mathematics Subject Classification. 46E35; 46E30, 42B15, $42 \mathrm{~B} 25$.

Key words and phrases. Non-standard growth, variable exponent, Besov space, Triebel-Lizorkin space, real interpolation, complex interpolation, trace operator, pseudodifferential operators. 
The main purpose of this paper is to present interpolation results in these new variable exponent scales. So far, complex interpolation of variable exponent Lebesgue spaces has been considered in $[11,12,26]$. Real interpolation, however, is more difficult in this setting. After some preliminaries, where we recall the definiton of the spaces we are dealing with and some auxiliary statements, in Section 3 we prove various real interpolation formulas for variable exponent Besov and Triebel-Lizorkin spaces. In particular, our results generalize some previous statements from $[9,29]$ where only the smoothness index was allowed to be variable. Real interpolation has subsequently also been studied by Kempka and Vybíral in the setting of variable exponent Lorentz spaces [25]. In [4] we and Harjulehto, Lukkari used real interpolation and weak variable exponent spaces to prove optimal regularity for measure valued $p(\cdot)$-Laplace PDEs.

Complex interpolation of variable exponent Besov spaces is discussed in Section 4. Finally, in Section 5 we give some applications of the interpolation results proved in the previous sections, namely we discuss a trace property for the variable index Besov spaces and the behavior of some pseudodifferential operators acting in these spaces.

Remark 1.1. Using standard notation, we have, for constant exponents,

$$
\left(L^{p_{0}}, L^{p_{1}}\right)_{\theta, q}=L^{p_{\theta}, q},
$$

where $1 / p_{\theta}:=\theta / p_{0}+(1-\theta) / p_{1}$ and $L^{p_{\theta}, q}$ is the Lorenz space. To obtain interpolation of Lebesgue spaces one simply chooses $q=p_{\theta}$. Although details have not been presented anywhere as best we know, it seems that there are no major difficulties in letting $p_{0}$ and $p_{1}$ be variable here, i.e.

$$
\left(L^{p_{0}(\cdot)}, L^{p_{1}(\cdot)}\right)_{\theta, q}=L^{p_{\theta}(\cdot), q},
$$

where $p_{\theta}$ is defined point-wise by the same formula as before. In this case the left hand side is the definition of the Lorentz space. One can show that it depends only on $p_{\theta}$ and $q$, not otherwise on the quantities on the left hand side.

However, this time we do not obtain an interpolation result in Lebesgue spaces, since we cannot set the constant $q$ equal to the function $p_{\theta}$. In fact, the role of $q$ in the real interpolation method is quite similar to the role of $q$ in the Besov space $B_{p, q}^{\alpha}$. Therefore, we hoped that the approach introduced in [3] for variable index Besov spaces would allow us to handle variable real exponent interpolation. Unfortunately, this seems not to be the case. Although we can define the interpolation functional $(\cdot, \cdot)_{\theta, q(\cdot)}$, it does not have the interpolation property. Therefore we now believe that this is not going to be a useful tool in the theory of variable exponent Lebesgue spaces. See also [25] for Lorentz spaces and [4] for an example of the usefulness of real interpolation in the variable setting.

Remark 1.2. We take this opportunity to correct a point in our paper [3]. In Theorem 8.1 we assumed the condition $\alpha^{-}>0$. However, the stronger assumption $\left(\alpha-\sigma_{p}\right)^{-}>0$ is needed for the claim to make sense directly. Although the stronger condition is not needed in the proof, the claim only holds with the weaker condition if the claim is appropriately interpreted. Consequently, the second sentence of Remark 8.3 is a truth with modification. We thank Hans-Gerd Leopold for this observation.

Moreover, we would like to clarify that in [3] we have used the fact that $\sum_{\nu \geqslant 0} \hat{\varphi}_{\nu}(\xi) \equiv 1$ to show the independence of the space $B_{p(\cdot), q(\cdot)}^{\alpha(\cdot)}$ with respect to the system $\left\{\varphi_{\nu}\right\}$ (cf. $[3$, Theorem 5.5]). It happens that this property does not necessarily follow from the assumptions in [3, Definition 5.1], and hence we should assume this additional condition on the system $\left\{\varphi_{\nu}\right\}$. This is not relevant when the Fourier transforms $\hat{\Phi}$ and $\hat{\varphi}$ are positive 
functions, since in that case one can find $c, C>0$ such that $c \leqslant \sum_{\nu \geqslant 0} \hat{\varphi}_{\nu}(\xi) \leqslant C$ for all $\xi \in \mathbb{R}^{n}$. Consequently, we can always use a normalized system in this situation. We thank António Caetano for a discussion where this question was posed.

\section{Preliminaries}

As usual, we denote by $\mathbb{R}^{n}$ the $n$-dimensional real Euclidean space, $\mathbb{N}$ the collection of all natural numbers and $\mathbb{N}_{0}=\mathbb{N} \cup\{0\}$. We write $B(x, r)$ for the open ball in $\mathbb{R}^{n}$ centered at $x \in \mathbb{R}^{n}$ with radius $r>0$. We use $c$ as a generic positive constant, i.e. a constant whose value may change from appearance to appearance. The expression $f \lesssim g$ means that $f \leqslant c g$ for some independent constant $c$, and $f \approx g$ means $f \lesssim g \lesssim f$.

The notation $X \hookrightarrow Y$ stands for continuous embeddings from $X$ to $Y$, where $X$ and $Y$ are quasi-normed spaces. If $E \subset \mathbb{R}^{n}$ is a measurable set, then $|E|$ stands for its (Lebesgue) measure and $\chi_{E}$ denotes its characteristic function. By supp $f$ we denote the support of the function $f$, i.e. the closure of its non-zero set.

The set $\mathcal{S}$ denotes the usual Schwartz class of rapidly decreasing complex-valued functions and $\mathcal{S}^{\prime}$ denotes the dual space of tempered distributions. The Fourier transform of a tempered distribution $f$ is denoted by $\hat{f}$.

2.1. Variable exponents. We denote by $\mathcal{P}_{0}$ the set of all measurable functions $p: \mathbb{R}^{n} \rightarrow$ $(0, \infty]$ (called variable exponents) which are bounded away from zero. The subset of those variable exponents with range $[1, \infty]$ is denoted by $\mathcal{P}$. For $A \subset \mathbb{R}^{n}$ and $p \in \mathcal{P}_{0}$ we denote $p_{A}^{+}=\operatorname{ess~sup}_{A} p(x)$ and $p_{A}^{-}=\operatorname{essinf}_{A} p(x)$; we abbreviate $p^{+}=p_{\mathbb{R}^{n}}^{+}$and $p^{-}=p_{\mathbb{R}^{n}}^{-}$.

Let

$$
\varphi_{p}(t)= \begin{cases}t^{p} & \text { if } p \in(0, \infty), \\ 0 & \text { if } p=\infty \text { and } t \leqslant 1 \\ \infty & \text { if } p=\infty \text { and } t>1\end{cases}
$$

The convention $1^{\infty}=0$ is adopted in order that $\varphi_{p}$ be left-continuous. In what follows we often write $t^{p}$ instead of $\varphi_{p}(t)$, with this convention implied. The variable exponent modular is defined by

$$
\varrho_{p(\cdot)}(f):=\int_{\mathbb{R}^{n}} \varphi_{p(x)}(|f(x)|) d x .
$$

The variable exponent Lebesgue space $L^{p(\cdot)}$ is the class of all measurable functions $f$ on $\mathbb{R}^{n}$ such that $\varrho_{p(\cdot)}(\lambda f)<\infty$ for some $\lambda>0$. This is a quasi-Banach space equipped with the quasi-norm

$$
\|f\|_{p(\cdot)}:=\inf \left\{\lambda>0: \varrho_{p(\cdot)}\left(\frac{1}{\lambda} f\right) \leqslant 1\right\} .
$$

If $p(x) \equiv p$ is constant, then $L^{p(\cdot)}=L^{p}$ is the classical Lebesgue space. More details on variable exponent Lebesgue spaces can be found in [11].

Variable exponent Sobolev spaces can then be defined from the $L^{p(\cdot)}$-space in the usual way.

We say that $g: \mathbb{R}^{n} \rightarrow \mathbb{R}$ is locally $\log$-Hölder continuous, abbreviated $g \in C_{\text {loc }}^{\text {log }}$, if there exists $c_{\log }>0$ such that

$$
|g(x)-g(y)| \leqslant \frac{c_{\log }}{\log (e+1 /|x-y|)}
$$


for all $x, y \in \mathbb{R}^{n}$. We say that $g$ is globally $\log$-Hölder continuous, abbreviated $g \in C^{\log }$, if it is locally log-Hölder continuous and there exists $g_{\infty} \in \mathbb{R}$ such that

$$
\left|g(x)-g_{\infty}\right| \leqslant \frac{c_{\log }}{\log (e+|x|)}
$$

for all $x \in \mathbb{R}^{n}$. The notation $\mathcal{P}^{\log }$ is used for those variable exponents $p \in \mathcal{P}$ with $\frac{1}{p} \in C^{\log }$. The class $\mathcal{P}_{0}^{\log }$ is defined analogously. If $p \in \mathcal{P}^{\log }$, then convolution with a radially decreasing $L^{1}$-function is bounded on $L^{p(\cdot)}$ :

$$
\|\varphi * f\|_{p(\cdot)} \leqslant c\|\varphi\|_{1}\|f\|_{p(\cdot)} .
$$

As in [13], we speak of $\eta$-functions meaning function on $\mathbb{R}^{n}$ of the form

$$
\eta_{\nu, m}(x):=\frac{2^{n \nu}}{\left(1+2^{\nu}|x|\right)^{m}}
$$

with $\nu \in \mathbb{N}$ and $m>0$. Note that $\eta_{\nu, m} \in L^{1}$ when $m>n$ and that $\left\|\eta_{\nu, m}\right\|_{1}=c_{m}$ is independent of $\nu$.

Let us recall some useful lemmas from [13].

Lemma 2.1 (Lemma 6.1, [13]). If $\alpha \in C_{\mathrm{loc}}^{\mathrm{log}}$, then there exists $d \in(n, \infty)$ such that if $m>d$, then

$$
2^{\nu \alpha(x)} \eta_{\nu, 2 m}(x-y) \leqslant c 2^{\nu \alpha(y)} \eta_{\nu, m}(x-y)
$$

with $c>0$ independent of $x, y \in \mathbb{R}^{n}$ and $\nu \in \mathbb{N}_{0}$.

The previous lemma allows us to treat the variable smoothness in many cases as if it were not variable at all, namely we can move the term inside the convolution as follows:

$$
2^{\nu \alpha(x)} \eta_{\nu, 2 m} * f(x) \leqslant c \eta_{\nu, m} *\left(2^{\nu \alpha(\cdot)} f\right)(x) .
$$

The next lemma often allows us to deal with exponents which are smaller than 1.

Lemma 2.2 ("The $r$-trick", Lemma A.7, [13]). Let $r>0, \nu \geqslant 0$ and $m>n$. Then there exists $c=c(r, m, n)>0$ such that

$$
|g(x)| \leqslant c\left(\eta_{\nu, m} *|g|^{r}(x)\right)^{1 / r}, \quad x \in \mathbb{R}^{n}
$$

for all $g \in \mathcal{S}^{\prime}$ with supp $\hat{g} \subset\left\{\xi:|\xi| \leqslant 2^{\nu+1}\right\}$

In order to deal with variable exponent Besov and Triebel-Lizorkin spaces we need to consider appropriate spaces of sequences of $L^{p(\cdot)}$-functions. The mixed Lebesgue-sequence space $L^{p(\cdot)}\left(\ell^{q(\cdot)}\right)$ can be easily defined with the constant exponent sequence space $\ell^{q(x)}$ for each point $x$, namely the (quasi)norm in this space is given by

$$
\left\|\left(f_{\nu}\right)_{\nu}\right\|_{L^{p(\cdot)}\left(\ell^{q(\cdot)}\right)}:=\|\|\left(f_{\nu}\right)_{\nu}\left\|_{\ell^{q(x)}}\right\|_{L^{p(\cdot)}} \text {. }
$$

This space was studied in [13]. The "opposite" case $\ell^{q(\cdot)}\left(L^{p(\cdot)}\right)$ is more complicated. The following definition is from [3].

Definition 2.3. Let $p, q \in \mathcal{P}_{0}$. The mixed sequence-Lebesgue space $\ell^{q(\cdot)}\left(L^{p(\cdot)}\right)$ is defined on sequences of $L^{p(\cdot)}$-functions by the modular

$$
\varrho_{\ell^{q(\cdot)}\left(L^{p(\cdot)}\right)}\left(\left(f_{\nu}\right)_{\nu}\right):=\sum_{\nu} \inf \left\{\lambda_{\nu}>0 \mid \varrho_{p(\cdot)}\left(f_{\nu} / \lambda_{\nu}^{\frac{1}{q(\cdot)}}\right) \leqslant 1\right\} .
$$


Here we use the convention $\lambda^{1 / \infty}=1$. The (quasi)norm is defined from this as usual:

$$
\left\|\left(f_{\nu}\right)_{\nu}\right\|_{\ell^{q(\cdot)}\left(L^{p(\cdot)}\right)}:=\inf \left\{\mu>0 \mid \varrho_{\ell^{q(\cdot)}\left(L^{p(\cdot)}\right)}\left(\frac{1}{\mu}\left(f_{\nu}\right)_{\nu}\right) \leqslant 1\right\} .
$$

Note that if $q^{+}<\infty$, then

$$
\inf \left\{\lambda>0 \mid \varrho_{p(\cdot)}\left(f / \lambda^{\frac{1}{q(\cdot)}}\right) \leqslant 1\right\}=\left\||f|^{q(\cdot)}\right\|_{\frac{p(\cdot)}{q(\cdot)}}
$$

In [3] we showed that $\|\cdot\|_{\ell^{q(\cdot)}\left(L^{p(\cdot)}\right)}$ is a norm when $q \geqslant 1$ is constant and $p(x) \geqslant 1$ or $\frac{1}{p(x)}+\frac{1}{q(x)} \leqslant 1$. More recently it was shown in [23] that $\|\cdot\|_{\ell^{q(\cdot)}\left(L^{p(\cdot)}\right)}$ is also a norm in the case $1 \leqslant q(x) \leqslant p(x) \leqslant \infty$; moreover, it is also shown that $\min \{p(x), q(x)\} \geqslant 1$ is not sufficient for it to be a norm.

It is worth noting that the Hardy-Littlewood maximal operator is not bounded in the spaces $L^{p(\cdot)}\left(\ell^{q(\cdot)}\right)$ and $\ell^{q(\cdot)}\left(L^{p(\cdot)}\right)$ for variable $q$. A way of overcoming this difficulty is to use convolution inequalities involving radially decreasing kernels, namely the $\eta$-functions defined above. The next statements often play the role of the boundedness of classical Fourier multipliers.

Theorem 2.4. Let $p, q \in \mathcal{P}^{\log }$.

(i) For $m>n,\left\|\left(\eta_{\nu, 2 m} * f_{\nu}\right)_{\nu}\right\|_{L^{p(\cdot)(\ell q(\cdot))}} \lesssim\left\|\left(f_{\nu}\right)_{\nu}\right\|_{\left.L^{p(\cdot)(\ell q(\cdot)}\right)} \quad\left(\right.$ with $\left.p^{+}, q^{+}<\infty\right)$. [13]

(ii) For sufficiently large $m,\left\|\left(\eta_{\nu, 2 m} * f_{\nu}\right)_{\nu}\right\|_{\ell^{q(\cdot)}\left(L^{p(\cdot)}\right)} \lesssim\left\|\left(f_{\nu}\right)_{\nu}\right\|_{\ell^{q(\cdot)}\left(L^{p(\cdot)}\right)} \cdot[3]$

Remark 2.5. In the case (ii) above the integer $m$ depends not only on $n$ but also on the constant involved in the log-Hölder continuity of $1 / q$. Moreover, the convolution inequality holds without the assumption $q \geqslant 1$. These facts were observed in [24] (cf. Lemma 10).

2.2. Besov and Triebel-Lizorkin spaces with variable indices. Besov and TriebelLizorkin spaces ( $B$ and $F$ spaces for short) with variable smoothness and integrability were recently introduced in [3] and [13], respectively. We recall here their definitions following the Fourier analytical approach. Let $(\varphi, \Phi)$ be a pair of functions in $\mathcal{S}$ satisfying

- $\operatorname{supp} \hat{\varphi} \subseteq\left\{\xi \in \mathbb{R}^{n}: \frac{1}{2} \leqslant|\xi| \leqslant 2\right\}$ and $|\hat{\varphi}(\xi)| \geqslant c>0$ when $\frac{3}{5} \leqslant|\xi| \leqslant \frac{5}{3}$,

- $\operatorname{supp} \hat{\Phi} \subseteq\left\{\xi \in \mathbb{R}^{n}:|\xi| \leqslant 2\right\}$ and $|\hat{\Phi}(\xi)| \geqslant c>0$ when $|\xi| \leqslant \frac{5}{3}$,

- $\sum_{\nu \geqslant 0} \hat{\varphi}_{\nu}(\xi)=1, \quad \xi \in \mathbb{R}^{n}$,

where $\varphi_{\nu}(x):=2^{\nu n} \varphi\left(2^{\nu} x\right)$ for $\nu \in \mathbb{N}$ and $\varphi_{0}(x):=\Phi(x)$. The system $\left\{\varphi_{\nu}\right\}$ is then said admissible. Fixing such a system, for measurable $\alpha: \mathbb{R}^{n} \rightarrow \mathbb{R}$ and $p, q \in \mathcal{P}_{0}$, the Besov space $B_{p(\cdot), q(\cdot)}^{\alpha(\cdot)}$ is defined as the class of all distributions $f \in \mathcal{S}^{\prime}$ such that

$$
\|f\|_{B_{p(\cdot), q(\cdot)}^{\alpha(\cdot)}}:=\left\|\left(2^{\nu \alpha(\cdot)} \varphi_{\nu} * f\right)_{\nu}\right\|_{\ell^{q(\cdot)}\left(L^{p(\cdot)}\right)}<\infty .
$$

The Triebel-Lizorkin space $F_{p(\cdot), q(\cdot)}^{\alpha(\cdot)}$ consists of all $f \in \mathcal{S}^{\prime}$ such that

$$
\|f\|_{F_{p(\cdot), q(\cdot)}^{\alpha(\cdot)}}:=\left\|\left(2^{\nu \alpha(\cdot)} \varphi_{\nu} * f\right)_{\nu}\right\|_{L^{p(\cdot)}\left(\ell^{q(\cdot)}\right)}<\infty .
$$

Notice that there is no difficulty in the $F$ space with $q$ depending on the space variable $x$ here, since the $\ell^{q(\cdot)}$-norm is inside the $L^{p(\cdot)}$-norm. As in the constant exponent case, they are quasi-Banach spaces and they agree when $p=q$, i.e., $B_{p(\cdot), p(\cdot)}^{\alpha(\cdot)}=F_{p(\cdot), p(\cdot)}^{\alpha(\cdot)}($ with $\alpha$ bounded).

These spaces are well-defined in the sense that different admissible systems produce the same spaces (up to equivalence of quasinorms). This is the case when $p, q \in \mathcal{P}_{0}^{\log }$ 
and $\alpha \in C_{\mathrm{loc}}^{\mathrm{log}}$, see [3, Theorem 5.5], [13, Theorem 3.10] (see also [21, Theorem 4.4, Lemma 2.5]). For simplicity, we thus omit the reference to the system $\left\{\varphi_{\nu}\right\}$ in the notation of the quasinorms.

The scale $F_{p(\cdot), q(\cdot)}^{\alpha}$ is rich enough to include Bessel potential spaces and Sobolev spaces with variable integrability as special cases. For $\alpha \in[0, \infty)$ and $p \in \mathcal{P}^{\log }$ with $1<p^{-} \leqslant$ $p^{+}<\infty$, it was proven in [12, Theorem 4.5] that $F_{p(\cdot), 2}^{\alpha}=\mathcal{L}^{\alpha, p(\cdot)}$ are the variable exponent Bessel potential spaces introduced in $[5,17]$. In particular, $\mathcal{L}^{k, p(\cdot)}=W^{k, p(\cdot)}$ are Sobolev spaces for $k \in \mathbb{N}_{0}$, under the same assumptions on $p$ [5, Corollary 6.2].

The Besov scale above includes variable order Hölder-Zygmund spaces as special cases (cf. [3, Theorem 7.2]). For $\alpha$ locally log-Hölder continuous with $\alpha^{-}>0$, we have

$$
B_{\infty, \infty}^{\alpha(\cdot)}=\mathcal{C}^{\alpha(\cdot)} \quad(\alpha \leqslant 1) \quad \text { and } \quad B_{\infty, \infty}^{\alpha(\cdot)}=C^{\alpha(\cdot)} \quad\left(\alpha^{+}<1\right) .
$$

We refer to $[3,6,7]$ for precise definitions of the spaces $\mathcal{C}^{\alpha(\cdot)}$ and $C^{\alpha(\cdot)}$.

The following embeddings will be useful below (see [3, Theorem 5.5]).

Proposition 2.6. Let $\alpha, \alpha_{0}, \alpha_{1} \in L^{\infty}$ and $p, q_{0}, q_{1} \in \mathcal{P}_{0}$.

(i) If $q_{0} \leqslant q_{1}$, then

(ii) If $\left(\alpha_{0}-\alpha_{1}\right)^{-}>0$, then

$$
B_{p(\cdot), q_{0}(\cdot)}^{\alpha(\cdot)} \hookrightarrow B_{p(\cdot), q_{1}(\cdot)}^{\alpha(\cdot)}
$$

$$
B_{p(\cdot), q_{0}(\cdot)}^{\alpha_{0}(\cdot)} \hookrightarrow B_{p(\cdot), q_{1}(\cdot)}^{\alpha_{1}(\cdot)}
$$

(iii) If $p^{+}, q^{+}<\infty$, then

$$
B_{p(\cdot), \min \{p(\cdot), q(\cdot)\}}^{\alpha(\cdot)} \hookrightarrow F_{p(\cdot), q(\cdot)}^{\alpha(\cdot)} \hookrightarrow B_{p(\cdot), \max \{p(\cdot), q(\cdot)\}}^{\alpha(\cdot)}
$$

Although one would obviously like to work in the variable index spaces independent of the choice of basis functions $\varphi_{\nu}$, the log-Hölder assumptions are quite strong in the sense that some results work under much weaker assumptions. By this reason, the conditions in Proposition 2.6 are those actually needed in the proof. Hence, these embeddings should be understood to hold when the same fixed set of basis functions is used for the definition of all spaces involved.

\section{REAL INTERPOLATION}

We recall that, for $0<\theta<1$ and $0<r \leqslant \infty$, the interpolation space $\left(A_{0}, A_{1}\right)_{\theta, r}$, formed from compatible quasi-normed spaces $A_{0}, A_{1}$, is the space of all elements $a \in A_{0}+A_{1}$ such that the quasi-norm

$$
\|a\|_{\left(A_{0}, A_{1}\right)_{\theta, r}}:=\left(\int_{0}^{\infty}\left[t^{-\theta} K(t, a)\right]^{r} \frac{d t}{t}\right)^{1 / r}
$$

(usual modification if $r=\infty$ ) is finite, where

$$
K(t, a)=K\left(t, a ; A_{0}, A_{1}\right)=\inf _{\substack{a_{0}+a_{1}=a \\ a_{0} \in A_{0}, a_{1} \in A_{1}}}\left(\left\|a_{0}\right\|_{A_{0}}+t\left\|a_{1}\right\|_{A_{1}}\right), \quad t>0,
$$

is the well-known Peetre $K$-functional.

For $p, q \in \mathcal{P}_{0}$ and $\alpha \in L^{\infty}$ we have $B_{p(\cdot), q(\cdot)}^{\alpha(\cdot)} \hookrightarrow \mathcal{S}^{\prime}[3$, Theorem 6.10]. Moreover, by Proposition 2.6 we also have $F_{p(\cdot), q(\cdot)}^{\alpha(\cdot)} \hookrightarrow \mathcal{S}^{\prime}$ (with $p^{+}<\infty$ ). Hence variable exponent $B$ and $F$ spaces form compatible couples of quasi-Banach spaces. We give some real interpolation results for these spaces. 
We refer to the monographs [8] and [40] for an exposition on interpolation theory.

Theorem 3.1. Let $0<\theta<1$ and $q \in(0, \infty]$. Moreover, let $p, q_{0}, q_{1} \in \mathcal{P}_{0}^{\log }$ and $\alpha_{0}, \alpha_{1} \in$

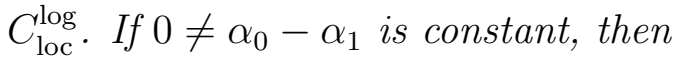

$$
\left(B_{p(\cdot), q_{0}(\cdot)}^{\alpha_{0}(\cdot)}, B_{p(\cdot), q_{1}(\cdot)}^{\alpha_{1}(\cdot)}\right)_{\theta, q}=B_{p(\cdot), q}^{\alpha(\cdot)}
$$

with $\alpha(x)=(1-\theta) \alpha_{0}(x)+\theta \alpha_{1}(x)$.

Proof. The general arguments below are similar to those used in [38, pp. 63-65], except the usage of Fourier multiplier statements, where we use convolution inequalities with $\eta$-functions instead. Nevertheless, we give some details for the reader's convenience. We only give the proof in the case $q \in(0, \infty)$, the case $q=\infty$ being treated by the same type of arguments up to usual modifications.

By symmetry we may assume $\alpha_{0}-\alpha_{1}>0$. Let $f=f_{0}+f_{1}$ with $f_{0} \in B_{p(\cdot), \infty}^{\alpha_{0}(\cdot)}$ and $f_{1} \in B_{p(\cdot), \infty}^{\alpha_{1}(\cdot)}$. Then

$\left\|2^{\nu \alpha_{0}(\cdot)} \varphi_{\nu} * f\right\|_{p(\cdot)} \lesssim\left\|2^{\nu \alpha_{0}(\cdot)} \varphi_{\nu} * f_{0}\right\|_{p(\cdot)}+\left\|2^{\nu \alpha_{0}(\cdot)} \varphi_{\nu} * f_{1}\right\|_{p(\cdot)} \lesssim\left\|f_{0}\right\|_{B_{p(\cdot), \infty}^{\alpha_{0}(\cdot)}}+2^{\nu\left(\alpha_{0}-\alpha_{1}\right)}\left\|f_{1}\right\|_{B_{p(\cdot), \infty}^{\alpha_{1}(\cdot)}}$.

Here we used that $\alpha_{0}-\alpha_{1}$ is constant. This implies that

$$
\left\|2^{\nu \alpha_{0}(\cdot)} \varphi_{\nu} * f\right\|_{p(\cdot)} \lesssim K\left(2^{\nu\left(\alpha_{0}-\alpha_{1}\right)}, f\right):=K\left(2^{\nu\left(\alpha_{0}-\alpha_{1}\right)}, f ; B_{p(\cdot), \infty}^{\alpha_{0}(\cdot)}, B_{p(\cdot), \infty}^{\alpha_{1}(\cdot)}\right) .
$$

Hence

$$
\|f\|_{B_{p(\cdot), q}^{\alpha(\cdot)}}^{q}=\sum_{\nu \geqslant 0} 2^{-\nu \theta\left(\alpha_{0}-\alpha_{1}\right) q}\left\|2^{\nu \alpha_{0}(\cdot)} \varphi_{\nu} * f\right\|_{p(\cdot)}^{q} \lesssim \sum_{\nu \geqslant 0} 2^{-\nu \theta\left(\alpha_{0}-\alpha_{1}\right) q} K\left(2^{\nu\left(\alpha_{0}-\alpha_{1}\right)}, f\right)^{q} .
$$

Using now the discretization

$$
(0, \infty)=\bigcup_{k \in \mathbb{Z}}\left[2^{(k-1)\left(\alpha_{0}-\alpha_{1}\right)}, 2^{k\left(\alpha_{0}-\alpha_{1}\right)}\right)
$$

and the fact that the functional $K(t, f)$ decreases with respect to $t$, we get

$$
\|f\|_{\left(\begin{array}{c}
\left.B_{p(\cdot), \infty}^{\alpha_{0}(\cdot)}, B_{p(\cdot), \infty}^{\alpha_{1}(\cdot)}\right)_{\theta, q} \\
q
\end{array}\right.}=\int_{0}^{\infty}\left[t^{-\theta} K(t, f)\right]^{q} \frac{d t}{t} \gtrsim \sum_{\nu \geqslant 0} K\left(2^{\nu\left(\alpha_{0}-\alpha_{1}\right)}, f\right)^{q} .
$$

Combining the two previous inequalities we obtain the embedding

$$
\left(B_{p(\cdot), \infty}^{\alpha_{0}(\cdot)}, B_{p(\cdot), \infty}^{\alpha_{1}(\cdot)}\right)_{\theta, q} \hookrightarrow B_{p(\cdot), q}^{\alpha(\cdot)}
$$

Let us show now that

$$
B_{p(\cdot), q}^{\alpha(\cdot)} \hookrightarrow\left(B_{p(\cdot), s}^{\alpha_{0}(\cdot)}, B_{p(\cdot), s}^{\alpha_{1}(\cdot)}\right)_{\theta, q} \quad \text { for } \quad 0<s<q .
$$

By Proposition 2.6 (ii) (note that $\alpha-\alpha_{1}=\left(\alpha_{0}-\alpha_{1}\right)(1-\theta)$ is a positive constant), we have

Let

$$
K(t, f)=K\left(t, f ; B_{p(\cdot), s}^{\alpha_{0}(\cdot)}, B_{p(\cdot), s}^{\alpha_{1}(\cdot)}\right) \lesssim t\|f\|_{B_{p(\cdot), q}^{\alpha(\cdot)}} .
$$

$$
\int_{0}^{\infty}\left[t^{-\theta} K(t, f)\right]^{q} \frac{d t}{t}=\int_{0}^{1}(\ldots)+\int_{1}^{\infty}(\ldots)=: I_{0}+I_{1} .
$$

Then (3.3) follows if we prove that both integrals $I_{0}$ and $I_{1}$ are bounded by $\|f\|_{B_{p(\cdot), q}^{\alpha(\cdot)}}^{q}$. This is clear for $I_{0}$ by the previous estimate. To estimate $I_{1}$, we use again that $\alpha_{0}-\alpha_{1}$ is a 
positive constant, and derive $I_{1} \lesssim \sum_{k \geqslant 0} 2^{-\theta k\left(\alpha_{0}-\alpha_{1}\right) q} K\left(2^{k\left(\alpha_{0}-\alpha_{1}\right)}, f\right)^{q}$. From an admissible system $\left\{\varphi_{\nu}\right\}$ we take the representation $f=f_{0}+f_{1}$ with

$$
f_{0}=\sum_{j=0}^{k} \varphi_{j} * f \quad \text { and } \quad f_{1}=\sum_{j \geqslant k+1} \varphi_{j} * f
$$

We can then prove that

$$
\left\|f_{0}\right\|_{B_{p(\cdot), s}^{\alpha_{0}(\cdot)}}^{s} \lesssim \sum_{\nu=0}^{k+1}\left\|2^{\nu \alpha_{0}(\cdot)} \varphi_{\nu} * f\right\|_{p(\cdot)}^{s} \quad \text { and } \quad\left\|f_{1}\right\|_{B_{p(\cdot), s}^{\alpha_{1}(\cdot)}}^{s} \lesssim \sum_{\nu \geqslant k}\left\|2^{\nu \alpha_{1}(\cdot)} \varphi_{\nu} * f\right\|_{p(\cdot)}^{s} .
$$

We postpone the proof of these inequalities for convenience. After straightforward calculations we then get

$$
\begin{aligned}
I_{1} & \lesssim \sum_{k \geqslant 0} 2^{k q\left(\alpha-\alpha_{0}\right)}\left[\sum_{\nu=0}^{k+1}\left\|2^{\nu \alpha_{0}(\cdot)} \varphi_{\nu} * f\right\|_{p(\cdot)}^{s}\right]^{q / s}+\sum_{k \geqslant 0} 2^{k q\left(\alpha-\alpha_{1}\right)}\left[\sum_{\nu \geqslant k}\left\|2^{\nu \alpha_{1}(\cdot)} \varphi_{\nu} * f\right\|_{p(\cdot)}^{s}\right]^{q / s} \\
& =: I_{1,1}+I_{1,2} .
\end{aligned}
$$

We next show that both quantities are dominated by $\|f\|_{B_{p(\cdot), q}^{\alpha(\cdot)}}^{q}$. Choose a measurable function $\beta_{0}: \mathbb{R}^{n} \rightarrow \mathbb{R}$ such that $\alpha(x)<\beta_{0}(x)<\alpha_{0}(x)$ and $\alpha_{0}-\beta_{0}$ is constant. An application of Hölder's inequality yields

$$
I_{1,1} \lesssim \sum_{k \geqslant 0} 2^{k q\left(\alpha-\alpha_{0}\right)}\left(\sum_{\nu=0}^{k+1} 2^{\nu \sigma\left(\alpha_{0}-\beta_{0}\right)}\right)^{q / \sigma}\left(\sum_{\nu=0}^{k+1}\left\|2^{\nu \beta_{0}(\cdot)} \varphi_{\nu} * f\right\|_{p(\cdot)}^{q}\right)
$$

with $\sigma=s(q / s)^{\prime}$. After calculating the middle sum and changing the summation order (noting that $\alpha-\beta_{0}<0$ is also constant), we obtain

$$
I_{1,1} \lesssim \sum_{\nu \geqslant 0} \sum_{k \geqslant \nu-1} 2^{(k-\nu)\left(\alpha-\beta_{0}\right) q}\left\|2^{\nu \alpha(\cdot)} \varphi_{\nu} * f\right\|_{p(\cdot)}^{q} \lesssim \sum_{\nu \geqslant 0}\left\|2^{\nu \alpha(\cdot)} \varphi_{\nu} * f\right\|_{p(\cdot)}^{q}
$$

The estimation of $I_{1,2}$ is similar (in this case take a function $\beta_{1}$ such that $\alpha_{1}(x)<\beta_{1}(x)<$ $\alpha(x)$ with $\alpha_{1}-\beta_{1}$ constant).

Using the monotonicity of interpolation, Proposition 2.6, and the embeddings (3.2) and (3.3) (with $0<s<\min \left\{q_{0}^{-}, q_{1}^{-}\right\}$), we obtain the announced interpolation formula.

Now we return to the estimates in (3.4). Both inequalities there can be proved in the same way, so that we treat only the second one. Fix $r \in\left(0, \min \left\{1, p^{-}\right\}\right)$and let $\left\{\psi_{\nu}\right\}$ an admissible system. Since $\hat{\psi}_{\nu} \hat{\varphi}_{j}=0$ when $|\nu-j|>1$, we have

$$
\left\|f_{1}\right\|_{B_{p(\cdot), s}^{\alpha_{1}(\cdot)}}^{s}=\sum_{\nu \geqslant k}\left\|2^{\nu \alpha_{1}(\cdot)} \sum_{\substack{|j-\nu| \leqslant 1 \\ j \geqslant k+1}} \psi_{\nu} * \varphi_{j} * f\right\|_{p(\cdot)}^{s} \lesssim \sum_{\nu \geqslant k} \sum_{j=-1}^{1}\left\|2^{\nu \alpha_{1}(\cdot) r}\left|\psi_{\nu} * \varphi_{\nu+j} * f\right|^{r}\right\|_{\frac{p_{p(\cdot)}^{r}}{r}}^{\frac{s}{r}} .
$$

Since $\left|\psi_{\nu}\right| \leqslant c \eta_{\nu, 2 m / r}$, with $c>0$ not depending on $\nu$, as in the proof of [3, Theorem 5.5] we use the $r$-trick (with $m>n$ large) and the Minkowski's integral inequality and obtain

$$
\left|\psi_{\nu} * \varphi_{\nu+j} * f\right|^{r} \lesssim \eta_{\nu+j, 2 m} *\left|\varphi_{\nu+j} * f\right|^{r} .
$$

This together with Lemma 2.1 and the boundedness of the convolution with $\eta$-functions in $L^{\frac{p(\cdot)}{r}}$, yields the estimate

$$
\left\|2^{\nu \alpha_{1}(\cdot) r}\left|\psi_{\nu} * \varphi_{\nu+j} * f\right|^{r}\right\|_{\frac{p(\cdot)}{r}}^{\frac{s}{r}} \lesssim\left\|2^{\nu \alpha_{1}(\cdot) r}\left|\varphi_{\nu+j} * f\right|^{r}\right\|_{\frac{p(\cdot)}{r}}^{\frac{s}{r}}=\left\|2^{\nu \alpha_{1}(\cdot)} \varphi_{\nu+j} * f\right\|_{p(\cdot)}^{s}
$$


and hence the estimate in (3.4) follows.

Remark 3.5. An interesting special case of Theorem 3.1 is the interpolation of variable order Hölder-Zygmund spaces. If $\alpha_{0}, \alpha_{1}$ are locally log-Hölder continuous with $0<\alpha_{0}^{-} \leqslant$ $\alpha_{0}(x)<\alpha_{1}(x) \leqslant \alpha_{1}^{+} \leqslant 1$ and $\alpha_{0}-\alpha_{1}$ constant, then

$$
\left(\mathcal{C}^{\alpha_{0}(\cdot)}, \mathcal{C}^{\alpha_{1}(\cdot)}\right)_{\theta, \infty}=\mathcal{C}^{\alpha(\cdot)}
$$

with $\alpha=(1-\theta) \alpha_{0}+\theta \alpha_{1}$. If, additionally, $\alpha_{1}^{+}<1$ then

$$
\left(C^{\alpha_{0}(\cdot)}, C^{\alpha_{1}(\cdot)}\right)_{\theta, \infty}=C^{\alpha(\cdot)} .
$$

Corollary 3.6. Let $0<\theta<1, q_{0}, q_{1} \in(0, \infty], p \in \mathcal{P}_{0}^{\log }$ and $\alpha \in C_{\mathrm{loc}}^{\log }$. Then

$$
\left(B_{p(\cdot), q_{0}}^{\alpha(\cdot)}, B_{p(\cdot), q_{1}}^{\alpha(\cdot)}\right)_{\theta, q}=B_{p(\cdot), q}^{\alpha(\cdot)} \quad \text { with } \quad \frac{1}{q}=\frac{1-\theta}{q_{0}}+\frac{\theta}{q_{1}}
$$

Proof. By Theorem 3.1 we can write $B_{p(\cdot), q_{i}}^{\alpha(\cdot)}=\left(B_{p(\cdot), 1}^{\alpha_{0}(\cdot)}, B_{p(\cdot), 1}^{\alpha_{1}(\cdot)}\right)_{\theta, q_{i}}, i=0,1$, where $\alpha_{0}, \alpha_{1} \in$ $C_{\mathrm{loc}}^{\mathrm{log}}$ are chosen in such a way that $\alpha_{0}-\alpha_{1}$ is constant and $(1-\theta) \alpha_{0}(x)+\theta \alpha_{1}(x)=$ $\alpha(x), x \in \mathbb{R}^{n}$. Hence the interpolation result follows from Theorem 3.1 and reiteration (see [8, Theorem 5.2.4]).

As for constant exponents, the next result shows that real interpolation between variable $F$ spaces with $p$ fixed always gives a variable $B$ space.

Corollary 3.7. Let $0<\theta<1$ and $q \in(0, \infty]$. Moreover, let $\alpha_{0}, \alpha_{1} \in C_{\mathrm{loc}}^{\log }, q_{0}, q_{1} \in \mathcal{P}_{0}$ and $p \in \mathcal{P}_{0}^{\log }$ with $p^{+}<\infty$. If $\alpha_{0}-\alpha_{1} \neq 0$ is a constant, then

$$
\left(B_{p(\cdot), q_{0}(\cdot)}^{\alpha_{0}(\cdot)}, F_{p(\cdot), q_{1}(\cdot)}^{\alpha_{1}(\cdot)}\right)_{\theta, q}=\left(F_{p(\cdot), q_{0}(\cdot)}^{\alpha_{0}(\cdot)}, F_{p(\cdot), q_{1}(\cdot)}^{\alpha_{1}(\cdot)}\right)_{\theta, q}=B_{p(\cdot), q}^{\alpha(\cdot)}
$$

with $\alpha(x)=(1-\theta) \alpha_{0}(x)+\theta \alpha_{1}(x)$.

Proof. Let $r \in\left(0, \min \left\{p^{-}, q_{0}^{-}, q_{1}^{-}\right\}\right)$. Then the monotonicity of interpolation couples and Proposition 2.6 yield

$$
\left(B_{p(\cdot), r}^{\alpha_{0}(\cdot)}, B_{p(\cdot), r}^{\alpha_{1}(\cdot)}\right)_{\theta, q} \hookrightarrow\left(F_{p(\cdot), q_{0}(\cdot)}^{\alpha_{0}(\cdot)}, F_{p(\cdot), q_{1}(\cdot)}^{\alpha_{1}(\cdot)}\right)_{\theta, q} \hookrightarrow\left(B_{p(\cdot), \infty}^{\alpha_{0}(\cdot)}, B_{p(\cdot), \infty}^{\alpha_{1}(\cdot)}\right)_{\theta, q} .
$$

Since the interpolation spaces on the left and right equal $B_{p(\cdot), q}^{\alpha(\cdot)}$ by Theorem 3.1 , the second equality of (3.8) follows. The other part is similar.

Recalling that $\mathcal{L}^{\alpha, p(\cdot)}=F_{p(\cdot), 2}^{\alpha}$ are Bessel potential spaces with variable integrability [13, Theorem 4.5] $\left(\alpha \geqslant 0\right.$ and $p \in \mathcal{P}^{\log }$ with $\left.1<p^{-} \leqslant p^{+}<\infty\right)$, which in turn are Sobolev spaces for integer $\alpha$, then formulas (3.8) include interesting special cases. In fact, we have

$$
\left(B_{p(\cdot), q_{0}(\cdot)}^{\alpha_{0}}, \mathcal{L}^{\alpha_{1}, p(\cdot)}\right)_{\theta, q}=\left(\mathcal{L}^{\alpha_{0}, p(\cdot)}, \mathcal{L}^{\alpha_{1}, p(\cdot)}\right)_{\theta, q}=B_{p(\cdot), q}^{\alpha},
$$

with $\alpha=(1-\theta) \alpha_{0}+\theta \alpha_{1}, \alpha_{0} \neq \alpha_{1}$, and $1<p^{-} \leqslant p^{+}<\infty$. In particular, for $\alpha_{i}=k_{i} \in \mathbb{N}_{0}$, $i=0,1$,

$$
\left(B_{p(\cdot), q_{0}(\cdot)}^{k_{0}}, W^{k_{1}, p(\cdot)}\right)_{\theta, q}=\left(W^{k_{0}, p(\cdot)}, W^{k_{1}, p(\cdot)}\right)_{\theta, q}=B_{p(\cdot), q}^{\alpha} \cdot
$$

Specializing further, we obtain that

$$
\left(B_{p(\cdot), q_{0}(\cdot)}^{\alpha}, L^{p(\cdot)}\right)_{\theta, q}=B_{p(\cdot), q}^{(1-\theta) \alpha} \quad \text { or, equivalently, } \quad\left(L^{p(\cdot)}, B_{p(\cdot), q_{0}(\cdot)}^{\alpha}\right)_{\theta, q}=B_{p(\cdot), q}^{\theta \alpha}
$$

for $\alpha \neq 0$. 


\section{Complex interpolation}

In the sequel the notation $[\cdot, \cdot]_{\theta}$ stands for the usual complex method of interpolation of Banach spaces (see $[8,40]$ ). Moreover, $\|\cdot\|_{\theta}$ denotes the corresponding norm in the interpolation space.

4.1. Interpolation of variable exponent Lebesgue spaces. Let $\omega$ be a positive and measurable function on $\mathbb{R}^{n}$. The weighted variable exponent Lebesgue space $L_{\omega}^{p(\cdot)}$ is defined as the collection of all measurable functions $f$ on $\mathbb{R}^{n}$ such that

$$
\|f\|_{L_{\omega}^{p(\cdot)}}:=\|f \omega\|_{L^{p(\cdot)}}<\infty .
$$

It is a Banach space equipped with this norm.

Complex interpolation of unweighted variable Lebesgue spaces was first studied in [12] (cf. [11, Chapter 7]). Recently some limiting cases of the exponent where treated in [26], generalizing to the variable exponent setting classical results on complex interpolation between Lebesgue spaces and the Hardy space $H^{1}$ or the space $B M O$. The following is the basic result for variable Lebesgue spaces.

Theorem 4.1 (Theorem 7.1.2, [11]). Let $0<\theta<1$ and $p_{0}, p_{1} \in \mathcal{P}$. Then

$$
\left[L_{\omega_{0}}^{p_{0}(\cdot)}, L_{\omega_{1}}^{p_{1}(\cdot)}\right]_{\theta}=L_{\omega}^{p_{\theta}(\cdot)} \text {, }
$$

where $\frac{1}{p_{\theta}(x)}=\frac{1-\theta}{p_{0}(x)}+\frac{\theta}{p_{1}(x)}$ and $\omega(x)=\omega_{0}(x)^{1-\theta} \omega_{1}(x)^{\theta}, \quad x \in \mathbb{R}^{n}$.

Moreover,

$$
\|\cdot\|_{\theta} \leqslant\|\cdot\|_{L_{\omega}^{p_{\theta}(\cdot)}} \leqslant 4\|\cdot\|_{\theta} .
$$

Formula (4.2) can be proved by adapting to the weighted case the steps given in [11, pp. 214-217] for the unweighted situation, which in turn follows the classical lines of [8]. For constant exponents formula (4.2) holds with equal norms. For general exponents we can not guarantee such equality.

From Theorem 4.1 we can deduce the following statement which extends to variable exponents a classical interpolation result of Stein and Weiss (cf. [8, Theorem 5.4.1]).

Corollary 4.3. Let $0<\theta<1$ and $p_{j}, q_{j} \in \mathcal{P}$ with $p_{j}^{+}, q_{j}^{+}<\infty, j=0,1$. Let $T$ be a linear operator which is bounded from $L_{\omega_{j}}^{p_{j}(\cdot)}$ into $L_{\nu_{j}}^{q_{j}(\cdot)}$. Then $T$ is also a bounded operator from $L_{\omega}^{p_{\theta}(\cdot)}$ into $L_{\nu}^{q_{\theta}(\cdot)}$, where $p_{\theta}, q_{\theta}, \omega, \nu$ are defined pointwise by

$$
\frac{1}{p_{\theta}}=\frac{1-\theta}{p_{0}}+\frac{\theta}{p_{1}} ; \quad \frac{1}{q_{\theta}}=\frac{1-\theta}{q_{0}}+\frac{\theta}{q_{1}} ; \quad \omega=\omega_{0}^{1-\theta} \omega_{1}^{\theta} ; \quad \nu=\nu_{0}^{1-\theta} \nu_{1}^{\theta} .
$$

Moreover,

$$
\|T\|_{L_{\omega}^{p_{\theta}(\cdot)} \rightarrow L_{\nu}^{q_{\theta}(\cdot)}} \leqslant 4\|T\|_{L_{\omega_{0}}^{p_{0}(\cdot)} \rightarrow L_{\nu_{0}}^{q_{0}(\cdot)}}\|T\|_{L_{\omega_{1}}^{p_{1}(\cdot) \rightarrow L_{\nu_{1}}^{q_{1}(\cdot)}}} .
$$

4.2. Interpolation of variable exponent Besov spaces. In this section we discuss complex interpolation in variable index Besov spaces. We base ourselves on the socalled retraction method which allows us to reduce the problem to the interpolation of appropriate sequence spaces. This well-known technique is described, for instance, in the monographs $[8,40]$. For the readers' convenience we recall that a Banach space $X$ is called a retract of a Banach space $Y$ if there are linear continuous operators $\mathfrak{R}: Y \rightarrow X$ (retraction) and $\mathfrak{J}: X \rightarrow Y$ (co-retraction) such that the composition $\mathfrak{R} \mathfrak{J}$ is the identity operator in $X$. 
Theorem 4.4. Let $\alpha \in C_{\mathrm{loc}}^{\log }, p \in \mathcal{P}^{\log }$ and $q \in(0, \infty]$. Then $B_{p(\cdot), q}^{\alpha(\cdot)}$ is a retract of $\ell^{q}\left(L_{2^{\nu \alpha(\cdot)}}^{p(\cdot)}\right)$.

In the proof of Theorem 4.4 we will use the following auxiliary result.

Lemma 4.5. Let $\alpha \in L^{\infty}$ and $p \in \mathcal{P}$. Suppose that $\left(g_{j}\right)_{j}$ is a sequence of tempered distributions such that

$\operatorname{supp} \hat{g}_{0} \subseteq\{\xi:|\xi| \leqslant 2\} \quad$ and $\quad \operatorname{supp} \hat{g}_{j} \subseteq\left\{\xi: 2^{j-1} \leqslant|\xi| \leqslant 2^{j+1}\right\}, \quad j \in \mathbb{N}$.

If $\left(g_{j}\right) \in \ell^{\infty}\left(L_{2^{j \alpha(\cdot)}}^{p(\cdot)}\right)$, then $\sum_{j \geqslant 0} g_{j}$ converges in $\mathcal{S}^{\prime}$.

Proof. We consider the case $\alpha^{-}<0$, the other case being simpler. Since $B_{\infty, \infty}^{-n-1+\alpha^{-}} \hookrightarrow \mathcal{S}^{\prime}$, it suffices to show that the sequence of partial sums $\left(\sum_{j=0}^{N} g_{j}\right)_{N \in \mathbb{N}}$ is a Cauchy sequence in $B_{\infty, \infty}^{-n-1+\alpha^{-}}$. We first note that

$$
\left\|\sum_{j=0}^{N} g_{j}-\sum_{j=0}^{M} g_{j}\right\|_{B_{\infty, \infty}^{-n-1+\alpha^{-}}} \leqslant \sum_{j=-2}^{2} \sum_{\nu=M-1}^{N+2} 2^{-\nu\left(n+1-\alpha^{-}\right)}\left\|\varphi_{\nu} * g_{\nu+j}\right\|_{\infty}, \quad N>M,
$$

which can be obtained from the assumptions on the supports of $\hat{\varphi}_{\nu}$ and $\hat{g}_{j}$. We show that

$$
\sum_{\nu \geqslant 0} 2^{-\nu\left(n+1-\alpha^{-}\right)}\left\|\varphi_{\nu} * g_{\nu+j}\right\|_{\infty}<\infty
$$

which clearly implies that the sequence of partial sums is Cauchy. Using that $\left|\varphi_{\nu}\right| \leqslant$ $\eta_{m, \nu} \leqslant 2^{n \nu} \eta_{m, 0}$, we obtain

$$
\begin{aligned}
2^{-\nu\left(n-\alpha^{-}\right)}\left\|\varphi_{\nu} * g_{\nu+j}\right\|_{\infty} & \leqslant \sup _{x} 2^{\nu \alpha^{-}} \int_{\mathbb{R}^{n}} \eta_{m, 0}(x-y)\left|g_{\nu+j}(y)\right| d y \\
& \leqslant \int_{\mathbb{R}^{n}} \eta_{m, 0}(x-y)^{p^{\prime}(y)}+\left(2^{\nu \alpha(y)}\left|g_{\nu+j}(y)\right|\right)^{p(y)} d y .
\end{aligned}
$$

Since $2^{\nu \alpha(\cdot)}\left|g_{\nu+j}\right| \in L^{p(\cdot)}$ uniformly (recall that $|j| \leqslant 2$ ), we see that the right-hand side is bounded provided $m>n$, which completes the proof.

Proof of Theorem 4.4. Let $\left(\varphi_{\nu}\right)_{\nu}$ be an admissible system and let $\chi_{j}:=\varphi_{j-1}+\varphi_{j}+\varphi_{j+1}$ (with $\varphi_{j} \equiv 0$ if $j<0$ ). As in the constant exponent case, we define

$$
\mathfrak{R}\left(f_{j}\right):=\sum_{j \geqslant 0} \chi_{j} * f_{j} \quad \text { and } \quad \mathfrak{J} f:=\left(\varphi_{\nu} * f\right) .
$$

Note that $\mathfrak{R}$ is well-defined by Lemma 4.5 since $\ell^{q}\left(L_{2^{\nu \alpha(\cdot)}}^{p(\cdot)}\right) \hookrightarrow \ell^{\infty}\left(L_{2^{\nu \alpha(\cdot)}}^{p(\cdot)}\right)$. Clearly $\mathfrak{J}$ is a bounded linear operator from $B_{p(\cdot), q}^{\alpha(\cdot)}$ into $\ell^{q}\left(L_{2^{\nu \alpha(\cdot)}}^{p(\cdot)}\right)$. Moreover, $\mathfrak{R J}$ is the identity map in $B_{p(\cdot), q}^{\alpha(\cdot)}$. So, it remains to show that $\Re$ is bounded from $\ell^{q}\left(L_{2^{\nu \alpha(\cdot)}}^{p(\cdot)}\right)$ into $B_{p(\cdot), q}^{\alpha(\cdot)}$. Taking into account the supports of $\hat{\varphi}_{\nu}$ and $\hat{\chi}_{j}$, we have

$$
\varphi_{\nu} * \mathfrak{R}\left(f_{j}\right)=\sum_{j=\nu-2}^{\nu+2} \varphi_{\nu} * \chi_{j} * f_{j}, \quad \nu=0,1, \ldots
$$


Estimating $\varphi_{\nu} * \varphi_{\nu+j+k} * f_{\nu+j}$ by a convolution with $\eta$-functions as in the last part of the proof of Theorem 3.1, we obtain

$$
\left\|\mathfrak{R}\left(f_{j}\right)\right\|_{B_{p(\cdot), q}^{\alpha(\cdot)}} \lesssim \sum_{j=-2}^{2} \sum_{k=-1}^{1}\|\| 2^{\nu \alpha(\cdot)} \varphi_{\nu} * \varphi_{\nu+j+k} * f_{\nu+j}\left\|_{L^{p(\cdot)}}\right\|_{\ell^{q}} \lesssim\|\| 2^{\nu \alpha(\cdot)} f_{\nu}\left\|_{L^{p(\cdot)}}\right\|_{\ell^{q}}
$$

We also need an interpolation result for general sequence spaces as follows. Let $\left(A_{\nu}\right)_{\nu}$ and $\left(B_{\nu}\right)_{\nu}$ be two sequences of Banach spaces such that $\left(A_{\nu}, B_{\nu}\right)$ form interpolation couples for any $\nu \in \mathbb{N}_{0}$. Then

$$
\left[\ell^{q_{0}}\left(A_{\nu}\right), \ell^{q_{1}}\left(B_{\nu}\right)\right]_{\theta}=\ell^{q}\left(\left[A_{\nu}, B_{\nu}\right]_{\theta}\right),
$$

where $0<\theta<1,1 \leqslant q_{0}, q_{1}<\infty$ and $1 / q=(1-\theta) / q_{0}+\theta / q_{1}$ [40, Section 1.18]. Here $\ell^{q}\left(A_{\nu}\right)$ denotes the (Banach) space of all sequences $a=\left(a_{\nu}\right)_{\nu}, a_{\nu} \in A_{\nu}$, such that $\|a\|_{\ell^{q}\left(A_{\nu}\right)}=\left(\sum_{\nu}\left\|a_{\nu}\right\|_{A_{\nu}}^{q}\right)^{1 / q}<\infty$.

Now we are ready to formulate the main statement on complex interpolation of variable Besov spaces.

Theorem 4.7. Let $0<\theta<1, \alpha_{0}, \alpha_{1} \in C_{\mathrm{loc}}^{\log }, q_{0}, q_{1} \in[1, \infty)$ and $p_{0}, p_{1} \in \mathcal{P}^{\log }$ with $1<p_{j}^{-} \leqslant p_{j}^{+}<\infty, j=0,1$. Then

where

$$
\left[B_{p_{0}(\cdot), q_{0}}^{\alpha_{0}(\cdot)}, B_{p_{1}(\cdot), q_{1}}^{\alpha_{1}(\cdot)}\right]_{\theta}=B_{p(\cdot), q}^{\alpha(\cdot)}
$$

$$
\alpha(x)=(1-\theta) \alpha_{0}(x)+\theta \alpha_{1}(x), \quad \frac{1}{p(x)}=\frac{1-\theta}{p_{0}(x)}+\frac{\theta}{p_{1}(x)} \quad \text { and } \quad \frac{1}{q}=\frac{1-\theta}{q_{0}}+\frac{\theta}{q_{1}} .
$$

Proof. The embedding into $\mathcal{S}^{\prime},\left[3\right.$, Theorem 6.10], shows that $\left\{B_{p_{0}(\cdot), q_{0}}^{\alpha_{0}(\cdot)}, B_{p_{1}(\cdot), q_{1}}^{\alpha_{1}(\cdot)}\right\}$ is an interpolation couple. Moreover, by Lemma 4.4

$$
\|f\|_{\left[B_{p_{0}(\cdot), q_{0}}^{\alpha_{0}(\cdot)}, B_{p_{1}(\cdot), q_{1}}^{\alpha_{1}(\cdot)}\right]_{\theta}} \approx\|\mathfrak{J} f\|_{\left[\ell q_{0}\left(L_{2^{\nu \alpha_{0}(\cdot)}}^{p_{0}(\cdot)}\right), \ell^{q_{1}}\left(L_{2^{\nu \alpha_{1}(\cdot)}}^{p_{1}(\cdot)}\right)\right]_{\theta}} .
$$

We then interpolate the sequence spaces on the right-hand side: by (4.6) and (4.2) we obtain

$$
\left[\ell^{q_{0}}\left(L_{2^{\nu \alpha_{0}(\cdot)}}^{p_{0}(\cdot)}\right), \ell^{q_{1}}\left(L_{2^{\nu \alpha_{1}(\cdot)}}^{p_{1}(\cdot)}\right)\right]_{\theta}=\ell^{q}\left(\left[L_{2^{\nu \alpha_{0}(\cdot)}}^{p_{0}(\cdot)}, L_{2^{\nu \alpha_{1}(\cdot)}}^{p_{1}(\cdot)}\right]_{\theta}\right)=\ell^{q}\left(L_{2^{\nu \alpha(\cdot)}}^{p(\cdot)}\right)
$$

\section{Applications}

In this section we give some applications of the interpolation results proved above.

5.1. Traces. We investigate the trace problem for the Besov spaces $B_{p(\cdot), q(\cdot)}^{\alpha(\cdot)}$ on the hyperplane $\mathbb{R}^{n-1}(n \geqslant 2)$. For constant exponents it is known that

$$
\operatorname{Tr} B_{p, q}^{\alpha}\left(\mathbb{R}^{n}\right)=B_{p, q}^{\alpha-\frac{1}{p}}\left(\mathbb{R}^{n-1}\right) \quad \text { and } \quad \operatorname{Tr} F_{p, q}^{\alpha}\left(\mathbb{R}^{n}\right)=F_{p, p}^{\alpha-\frac{1}{p}}\left(\mathbb{R}^{n-1}\right)=B_{p, p}^{\alpha-\frac{1}{p}}\left(\mathbb{R}^{n-1}\right)
$$

if $\alpha>\frac{1}{p}+(n-1) \max \left\{0, \frac{1}{p}-1\right\}$ (with $p<\infty$ in the $F$ space). We refer to [38, Sect. 2.7.2] for further details and historical remarks.

The corresponding problem for the variable exponent Triebel-Lizorkin scale was studied in [13]. If $p, q \in \mathcal{P}_{0}^{\log }$, with $p^{+}, q^{+}<\infty$, and $\alpha \in C_{\mathrm{loc}}^{\log }$ has a limit at infinity, then

$$
\operatorname{Tr} F_{p(\cdot), q(\cdot)}^{\alpha(\cdot)}\left(\mathbb{R}^{n}\right)=F_{p(\cdot), p(\cdot)}^{\alpha(\cdot)-\frac{1}{p(\cdot)}}\left(\mathbb{R}^{n-1}\right) \quad \text { if } \quad\left(\alpha-\frac{1}{p}-(n-1) \max \left\{0, \frac{1}{p}-1\right\}\right)^{-}>0
$$


Remark 5.2. The proof given in [13, Theorem 3.13] is incomplete: in fact, only the inclusion $\operatorname{Tr} F_{p(\cdot), q(\cdot)}^{\alpha(\cdot)}\left(\mathbb{R}^{n}\right) \hookrightarrow F_{p(\cdot), p(\cdot)}^{\alpha(\cdot)-\frac{1}{p(\cdot)}}\left(\mathbb{R}^{n-1}\right)$ is shown. However, the opposite (easier) inclusion is proved as in the classical case, see [16].

For the variable Besov spaces we have the following result (for constant $q$ ).

Theorem 5.3. Let $p \in \mathcal{P}_{0}^{\log }$ with $p^{+}<\infty, q \in(0, \infty]$ and $\alpha \in C_{\mathrm{loc}}^{\log }$ with limit at infinity. If $\left(\alpha-\frac{1}{p}-(n-1) \max \left\{0, \frac{1}{p}-1\right\}\right)^{-}>0$, then

$$
\operatorname{Tr} B_{p(\cdot), q}^{\alpha(\cdot)}\left(\mathbb{R}^{n}\right)=B_{p(\cdot), q}^{\alpha(\cdot)-\frac{1}{p(\cdot)}}\left(\mathbb{R}^{n-1}\right) .
$$

Proof. Suppose first that all exponents are defined on $\mathbb{R}^{n}$. Take a function $\alpha_{0} \in C_{\text {loc }}^{\text {log }}$, with limit at infinity, such that $\alpha-\alpha_{0}$ is a positive constant and $\left(\alpha_{0}-\frac{1}{p}-(n-1) \max \left\{0, \frac{1}{p}-\right.\right.$ $1\})^{-}>0$. Choose now $\alpha_{1}:=2 \alpha-\alpha_{0}$. Since $\left(\alpha_{1}-\frac{1}{p}-(n-1) \max \left\{0, \frac{1}{p}-1\right\}\right)^{-}>0$, by (5.1) we have

$$
\operatorname{Tr} F_{p(\cdot), q}^{\alpha_{j}(\cdot)}\left(\mathbb{R}^{n}\right)=F_{p(\cdot), p(\cdot)}^{\alpha_{j}(\cdot)-\frac{1}{p(\cdot)}}\left(\mathbb{R}^{n-1}\right)
$$

for $j=0,1$. Since $\alpha_{1}-\alpha_{0}$ is also a positive constant and $\frac{1}{2}\left(\alpha_{0}+\alpha_{1}\right)=\alpha$, the result follows by interpolation: indeed, by Corollary 3.7 we have

$$
B_{p(\cdot), q}^{\alpha(\cdot)}\left(\mathbb{R}^{n}\right)=\left(F_{p(\cdot), 1}^{\alpha_{0}(\cdot)}\left(\mathbb{R}^{n}\right), F_{p(\cdot), 1}^{\alpha_{1}(\cdot)}\left(\mathbb{R}^{n}\right)\right)_{\frac{1}{2}, q}
$$

and

$$
B_{p(\cdot), q}^{\alpha(\cdot)-\frac{1}{p(\cdot)}}\left(\mathbb{R}^{n-1}\right)=\left(F_{p(\cdot), p(\cdot)}^{\alpha_{0}(\cdot)-\frac{1}{p(\cdot)}}\left(\mathbb{R}^{n-1}\right),\left(F_{p(\cdot), p(\cdot)}^{\alpha_{1}(\cdot)-\frac{1}{p(\cdot)}}\left(\mathbb{R}^{n-1}\right)\right)_{\frac{1}{2}, q}\right.
$$

Hence by the interpolation property, we conclude that the trace operator maps $B_{p(\cdot), q}^{\alpha(\cdot)}\left(\mathbb{R}^{n}\right)$ into $B_{p(\cdot), q}^{\alpha(\cdot)-\frac{1}{p(\cdot)}}\left(\mathbb{R}^{n-1}\right)$ boundedly.

If we are proving the inclusion " $\supset$ ", then the exponents are, a priori, only defined on $\mathbb{R}^{n-1}$. However, in this case they can be extended to whole $\mathbb{R}^{n}$ with the same log-Hölder constants by [11, Proposition 4.1.7].

5.2. Pseudodifferential operators. The aim of this section is to study mapping properties of pseudodifferential operators in variable exponent Besov spaces. Some standard references for such operators are [20,36, 37, 39].

For $\mu \in \mathbb{R}, 0 \leqslant \delta \leqslant 1$, let $S_{1, \delta}^{\mu}$ be the Hörmander class of complex-valued $C^{\infty}$-functions $a=a(x, \xi)$ in $\mathbb{R}^{n} \times \mathbb{R}^{n}$ such that for all multi-indices $\gamma, \beta$ there exists a positive constant $c_{\gamma, \beta}$ with

$$
\left|D_{x}^{\gamma} D_{\xi}^{\beta} a(x, \xi)\right| \leqslant c_{\gamma, \beta}\langle\xi\rangle^{\mu-|\beta|+\delta|\gamma|}
$$

for all $x, \xi \in \mathbb{R}^{n}$, where we use the abbreviations

$$
\langle\xi\rangle=\left(1+|\xi|^{2}\right)^{1 / 2}, \quad D_{x}^{\gamma}=\frac{\partial^{|\gamma|}}{\partial x_{1}^{\gamma_{1}} \cdots \partial x_{n}^{\gamma_{n}}} \quad \text { and } \quad D_{\xi}^{\beta}=\frac{\partial^{|\beta|}}{\partial \xi_{1}^{\beta_{1}} \cdots \partial \xi_{n}^{\beta_{n}}} .
$$

With a symbol $a \in S_{1, \delta}^{\mu}$ we associate the pseudodifferential operator $a(x, D)$ defined on the Schwartz class $\mathcal{S}$ by

$$
a(x, D) f(x)=\int_{\mathbb{R}^{n}} e^{\mathrm{i} x \cdot \xi} a(x, \xi) \widehat{f}(\xi) d \xi .
$$

It is known that pseudodifferential operators with symbols belonging to $S_{1, \delta}^{\mu}, \mu \in \mathbb{R}$, $0 \leqslant \delta<1$, are bounded from $B_{p, q}^{\alpha}$ into $B_{p, q}^{\alpha-\mu}$ for any (constant) exponents $\alpha \in \mathbb{R}$, 
$0<p, q \leqslant \infty$, and a similar assertion holds for Triebel-Lizorkin spaces (under the additional assumption $p<\infty$ ) (see e.g. [39, Chapter 6] for further details).

Recently, Rabinovich and Samko [33] proved the boundedness of some pseudodifferential operators in weighted variable exponent Lebesgue and Bessel potential spaces. The following is contained in [33, Theorem 5.1].

Theorem 5.4. Let $\alpha \in \mathbb{R}$ and $p \in \mathcal{P}^{\log }$ with $1<p^{-} \leqslant p^{+}<\infty$. If $a \in S_{1,0}^{\mu}(\mu \in \mathbb{R})$, then the operator $a(x, D)$ is bounded from $\mathcal{L}^{\alpha, p(\cdot)}$ into $\mathcal{L}^{\alpha-\mu, p(\cdot)}$.

Taking into account Theorem 5.4 and observing that Besov spaces can be written as a (real) interpolation space between appropriate Bessel potential spaces, cf. (3.9), we deduce the following result.

Theorem 5.5. Let $\alpha \in \mathbb{R}, p \in \mathcal{P}^{\log }$ with $1<p^{-} \leqslant p^{+}<\infty$, and $0<q \leqslant \infty$. Then any pseudodifferential operator $a(x, D)$ with a symbol $a \in S_{1,0}^{\mu}(\mu \in \mathbb{R})$ is bounded from $B_{p(\cdot), q}^{\alpha}$ into $B_{p(\cdot), q}^{\alpha-\mu}$.

\section{REFERENCES}

[1] E. Acerbi and G. Mingione: Regularity results for a class of functionals with nonstandard growth, Arch. Ration. Mech. Anal. 156 (2001), no. 2, 121-140.

[2] E. Acerbi and G. Mingione: Gradient estimates for the $p(x)$-Laplacian system, J. Reine Angew. Math. 584 (2005), 117-148.

[3] A. Almeida and P. Hästö: Besov spaces with variable smoothness and integrability, J. Funct. Anal. 258 (2010), no. 5, 1628-1655.

[4] A. Almeida, P. Harjulehto, P. Hästö and T. Lukkari: Riesz and Wolff potentials and elliptic equations in variable exponent weak Lebesgue spaces, Preprint (2013).

[5] A. Almeida and S. Samko: Characterization of Riesz and Bessel potentials on variable Lebesgue spaces, J. Function Spaces Appl. 4 (2006), no. 2, 113-144.

[6] A. Almeida and S. Samko: Pointwise inequalities in variable Sobolev spaces and applications, $Z$. Anal. Anwend. 26 (2007), no. 2, 179-193.

[7] A. Almeida and S. Samko: Embeddings of variable Hajłasz-Sobolev spaces into Hölder spaces of variable order, J. Math. Anal. Appl. 353 (2009), no. 2, 489-496.

[8] J. Bergh and J. Löfström: Interpolation Spaces. An introduction, Springer-Verlag, Berlin, 1976.

[9] O. Besov: Interpolation, embedding, and extension of spaces of functions of variable smoothness, (Russian) Tr. Mat. Inst. Steklova 248 (2005), Issled. po Teor. Funkts. i Differ. Uravn., 52-63. [Translation in Proc. Steklov Inst. Math. 248 (2005), no. 1, 47-58.]

[10] Y. Chen, S. Levine and R. Rao: Variable exponent, linear growth functionals in image restoration, SIAM J. Appl. Math. 66 (2006), no. 4, 1383-1406.

[11] L. Diening, P. Harjulehto, P. Hästö and M. Růžička: Lebesgue and Sobolev Spaces with Variable Exponents, Lecture Notes in Mathematics, vol. 2017, Springer-Verlag, Berlin, 2011.

[12] L. Diening, P. Hästö and A. Nekvinda: Open problems in variable exponent Lebesgue and Sobolev spaces, FSDONA04 Proceedings (Drabek and Rákosník (eds.); Milovy, Czech Republic, 2004), 3858.

[13] L. Diening, P. Hästö and S. Roudenko: Function spaces of variable smoothness and integrability, J. Funct. Anal. 256 (2009), no. 6, 1731-1768.

[14] X.-L. Fan: Global $C^{1, \alpha}$ regularity for variable exponent elliptic equations in divergence form, $J$. Differential Equations 235 (2007), no. 2, 397-417.

[15] R. Fortini, D. Mugnai and P. Pucci: Maximum principles for anisotropic elliptic inequalities, Nonlinear Anal. 70 (2009), no. 8, 2917-2929.

[16] M. Frazier and B. Jawerth: Decomposition of Besov spaces, Indiana Univ. Math. J. 34 (1985), $777-799$.

[17] P. Gurka, P. Harjulehto and A. Nekvinda: Bessel potential spaces with variable exponent, Math. Inequal. Appl. 10 (2007), no. 3, 661-676. 
[18] P. Harjulehto, P. Hästö, V. Latvala, O. Toivanen: Gamma convergence for functionals related to image restoration, Appl. Math. Letters 26 (2013), 56-60.

[19] P. Harjulehto, P. Hästö, U. Lê and M. Nuortio: Overview of differential equations with non-standard growth, Nonlinear Anal. 72 (2010), no. 12, 4551-4574.

[20] L. Hörmander: The Analysis of Linear Partial Differential Operators, III, IV, Springer, New York, 1985.

[21] H. Kempka: 2-microlocal Besov and Triebel-Lizorkin spaces of variable integrability, Rev. Mat. Complut. 22 (2009), no. 1, 227-251.

[22] H. Kempka: Atomic, molecular and wavelet decomposition of 2-microlocal Besov and TriebelLizorkin spaces with variable integrability, Funct. Approx. Comment. Math. 43 (2010), part 2, 171-208.

[23] H. Kempka and J. Vybíral: A note on the spaces of variable integrability and summability of Almeida and Hästö, Proc. Amer. Math. Soc. 141 (2013), no. 9, 3207-3212.

[24] H. Kempka and J. Vybíral: Spaces of variable smoothness and integrability: Characterizations by local means and ball means of differences, J. Fourier Anal. Appl. 18 (2012), no. 4, 852-891.

[25] H. Kempka and J. Vybíral: Lorentz spaces with variable exponents, Math. Nachr., to appear.

[26] T. Kopaliani: Interpolation theorems for variable exponent Lebesgue spaces, J. Funct. Anal. 257 (2009), no. 11, 3541-3551.

[27] O. Kováčik and J. Rákosník: On spaces $L^{p(x)}$ and $W^{1, p(x)}$, Czechoslovak Math. J. 41(116) (1991), 592-618.

[28] H.-G. Leopold: On Besov spaces of variable order of differentiation, Z. Anal. Anwendungen 8 (1989), no. $1,69-82$.

[29] H.-G. Leopold: Interpolation of Besov spaces of variable order of differentiation, Arch. Math. 53 (1989), 178-187.

[30] H.-G. Leopold: On function spaces of variable order of differentiation, Forum Math. 3 (1991), 633644.

[31] F. Li, Z. Li and L. Pi: Variable exponent functionals in image restoration, Appl. Math. Comput. 216 (2010), no. 3, 870-882.

[32] W. Orlicz: Über konjugierte Exponentenfolgen, Studia Math. 3 (1931), 200-212.

[33] V. Rabinovich and S. Samko: Boundedness and Fredholmness of pseudodifferential operators in variable exponent spaces, Integral Equations Operator Theory 60 (2008), no. 4, 507-537.

[34] M. Růžička: Electrorheological fluids: modeling and mathematical theory, Lecture Notes in Mathematics, 1748, Springer-Verlag, Berlin, 2000.

[35] R. Schneider, O. Reichmann and C. Schwab: Wavelet solution of variable order pseudodifferential equations, Calcolo 47 (2010), no. 2, 65-101.

[36] M.E. Taylor: Partial Differential Equations II, Applied Mathematical Sciences, vol. 116, Springer, New York, 1996.

[37] M.E. Taylor: Tools for PDE: Pseudodifferential Operators, Paradifferential Operators and Layer Potentials, Mathematical Surveys and Monographs, vol. 81, AMS, Providence, 2000.

[38] H. Triebel: Theory of Function Spaces, Monographs in Mathematics 78, Birkhäuser Verlag, Basel, 1983.

[39] H. Triebel: Theory of Function Spaces II, Monographs in Mathematics 84, Birkhäuser Verlag, Basel, 1992.

[40] H. Triebel: Interpolation Theory, Function Spaces, Differential Operators (2nd ed.), Johann Ambrosius Barth, Heidelberg, 1995.

[41] J. Vybíral: Sobolev and Jawerth embeddings for spaces with variable smoothness and integrability, Ann. Acad. Sci. Fenn. Math. 34 (2009), no. 2, 529-544.

[42] J.-S. Xu: Variable Besov and Triebel-Lizorkin spaces, Ann. Acad. Sci. Fenn. Math. 33 (2008), $511-522$.

[43] J.-S. Xu: The relation between variable Bessel potential spaces and Triebel-Lizorkin spaces, Integral Transforms Spec. Funct. 19 (2008), no. 8, 599-605. 
Department of Mathematics, University of Aveiro, 3810-322 Aveiro, Portugal E-mail address: jaralmeida@ua.pt

Department of Mathematical Sciences, P.O. Box 3000, Fi-90014 University of Oulu, FINLAND

E-mail address: peter.hasto@helsinki.fi

URL: http://cc.oulu.fi/ phasto/ 